\title{
Genetic structure of black coral populations in New Zealand's fiords
}

\author{
K. J. Miller* \\ National Institute of Water and Atmospheric Research Ltd, PO Box 14-901, Kilbirnie, Wellington, New Zealand
}

\begin{abstract}
The antipatharian black coral Antipathes fiordensis is endemic to south-western New Zealand, where most of the population biomass occurs in shallow water throughout a network of fiords and inlets. The planulae larvae of $A$. fiordensis are negatively buoyant, weak swimming and short lived. These factors, coupled with hydrographic observations of restricted water movement in the fiords, suggest that larval dispersal and gene flow will be limited in this species, and that separate fiords may represent genetically isolated populations. This study tests the hypothesis of limited larval dispersal in $A$. fiordensis by examining the genetic variation and population subdivision both within and among fiords. Allozyme electrophoresis at 10 polymorphic loci revealed an unusual population genetic structure in A. fiordensis. Significant genetic variation was found among sites (mean $F_{S T}=$ $0.046, \mathrm{p}<0.01$ ), suggesting larval dispersal is restricted even at distances of 10 to $15 \mathrm{~km}$. Interestingly, most genetic differentiation occurred between sites within fiords $\left(F_{S T}=0.034\right)$, rather than among fiords $\left(F_{S i}=0.02\right)$ which is contrary to the pattern expected if the fiord populations were reproductively isolated. Furthermore, regression of gene flow with geographic distance showed no evidence of isolationby-distance between populations $\left(r^{2}=0.017\right)$, as might be expected in a species with limited larval dispersal. Asexual reproduction was apparent in populations of A. fiordensis, as evidenced by genotypic diversity ratios $<1$, as well as significant departures from random mating associated with a combination of heterozygote deficits and excesses at the majority of sites. I propose that the atypical genetic structure observed for $A$. fiordensis in Fiordland represents a population that has not yet reached equilibrium due to a combination of the effects of recent colonisation, asexual reproduction and the potential longevity of individual coral genotypes.
\end{abstract}

KEY WORDS: Antipatharian corals - Gene flow · Population genetics - Asexual reproduction - Larval dispersal

\section{INTRODUCTION}

The south-western coast of New Zealand is characterised by a series of deep water fiords that were carved out by glaciers during the last ice age. The fiords are deep (over $400 \mathrm{~m}$ at the deepest point), narrow, steep sided basins which are separated from coastal waters by submarine sills. The phenomenal rainfall in Fiordland (up to $7000 \mathrm{~mm}$ annually) causes the build up of a tannin-laden freshwater surface layer within the fiords. The freshwater layer, which is about $5 \mathrm{~m}$ thick on average, absorbs light and significantly reduces incident light levels in the seawater below.

•E-mail:k.miller@niwa.cri.nz
Consequently, the dark, calm fiord environments allow many marine species which are usually restricted to deep water $(>100 \mathrm{~m})$ to flourish in shallow water $(<50 \mathrm{~m})$. These deep-water emergent species include antipatharian corals, stylasteriniid hydrocorals, hexactinellid sponges and several fish species (Grange et al. 1981).

Circulation patterns within the fiords are estuarine. The freshwater surface layer flows seawards, entraining seawater with it, and the outflowing water is then replaced by a deeper inflow of dense, saline ocean water (Stanton \& Pickard 1981, Stanton 1984). The estuarine flows within the fiords will have important consequences for benthic marine species which rely on planktonic larvae for dispersal. Because the net flow of 
seawater is inwards, it has generally been assumed that larvae will be retained within natal fiords and that there will be no larval dispersal between the 14 separate fiords which span over $200 \mathrm{~km}$ of the New Zealand coastline. If each of the fiords acts as a closed system, then the fiord populations wil] be excellent natural laboratories to study the effects of isolation and natural selection in marine species.

The endemic black coral Antipathes fiordensis (Grange 1990) is widespread, conspicuous and numerically abundant throughout the fiords, with the total population estimated to number over 7 million colonies (Grange 1985). A small population of A. fiordensis has also been reported from shallow water in Port Pegasus, Stewart Island, over $100 \mathrm{~km}$ south of the Fiordland region (Grange 1994). Like many corals (Harrison \& Wallace 1990), A. fiordensis produces planulae larvae which are thought to facilitate dispersal and the colonisation of new habitat. Sexual reproduction in A. fiordensis occurs annually (Grange 1988, Grange \& Singleton 1988) and larvae appear to be negatively buoyant, non-feeding and short lived (approximately $10 \mathrm{~d}$; Miller 1996). These factors, in combination with the estuarine circulation patterns within the fiords, suggest that larval dispersal of $A$. fiordensis will be restricted. This in turn is expected to reduce gene flow and influence the genetic structure of populations (Jackson 1986).

Typically, in a benthic species, limited planktonic dispersal will result in subdivided populations due to effects such as inbreeding (Knowlton \& Jackson 1993), random genetic drift and localised selection (Burton 1986, Jackson 1986). In an earlier study (Miller \& Grange in press) it was shown that significant levels of genetic variation occur among Antipathes fiordensis populations at 7 sites within a single fiord (Doubtful Sound). This is consistent with the hypothesised population structure resulting from restricted larval dispersal. However, contrary to expectations, comparisons with a second fiord, Nancy Sound, suggested there may be surprisingly little genetic differentiation between individual fiords (Miller \& Grange in press).

Earlier studies also identified black coral colonies with identical genotypes (Miller \& Grange in press), indicating asexual reproduction may play an important part in the life history of Antipathes fiordensis. A type of polyp 'bail-out' has been observed in $A$. fiordensis (Miller \& Grange in press), although the timing, frequency and extent of asexual reproduction in $A$. fiordensis remains unclear. The presence of clones at multiple sites is thought to reflect wider dispersal capabilities in asexually produced larvae than in sexually produced larvae (Miller \& Grange in press). If true, then dispersal strategies in $A$. fiordensis are contrary to the widely held ecological theories which predict that asexual reproduction will maintain local populations while sexual reproduction will facilitate gene flow and the colonisation of new habitats (e.g Stoddart 1984 . Johnson \& Threlfall 1987, Ayre \& Willis 1988).

In this study, I investigated the genetic structure of A. fiordensis populations from an additional 11 fiords throughout the Fiordland region in order to (1) address levels of connectivity between separate fiords along the New Zealand south-western coastline, specifically to determine levels of gene flow both within and between fiords and (2) to determine the relative contributions of sexual and asexual reproduction to recruitment within each of the fiords.

\section{MATERIALS AND METHODS}

Sample collection. Samples of Antipathes fiordensis were collected from a total of 27 sites in 13 fiords throughout Fiordland, south-western New Zealand, and from 1 site at Stewart Island, approximately $112 \mathrm{~km}$ south of Fiordland (Fig. 1). Replicate sites within each fiord were separated by approximately 10 to $15 \mathrm{~km}$, and the depth distribution of sampled colonies was between 15 and $35 \mathrm{~m}$. Tissue samples of corals were collected by removing 50 to $100 \mathrm{~mm}$ long branch tips from approximately 30 colonies at each site, taking care to avoid contamination of samples by epibionts. Samples were placed in individual bags underwater and were transferred to liquid nitrogen immediately on reaching the surface.

Electrophoresis. Allozyme electrophoresis was used to determine the 12-locus genotypes of the 734 Antipathes fiordensis colonies collected, in order to compare the genetic structure of black coral populations within and between fiords. Starch gels (12\% w/v) were stained for 9 different enzymes: malate dehydrogenase $(\mathrm{MDH})$, glucose-phosphate isomerase (GPI), phosphoglucomutase (PGM), hexokinase (HK, 2 loci), malic enzyme (ME), esterase (EST, 2 loci), isocitric dehydrogenase (IDH), leucyl-alanine-peptidase (LA, 2 loci) and xanthine oxidase (XO). Details of running conditions and staining methods can be found in Miller \& Grange (in press).

Statistical analysis. Allelic frequencies and population genetic statistics were calculated using BIOSYS (Swofford \& Selander 1981). In these analyses each genotype was included only once at each site. Exact tests (Elston \& Forthofer 1977) were used to test for departures from random mating within each of the populations as expected under conditions of HardyWeinberg equilibrium. Rare alleles were pooled for these tests and the probability of significance was corrected for multiple simultaneous tests to reduce the chance of Type 1 errors (Miller 1966). 


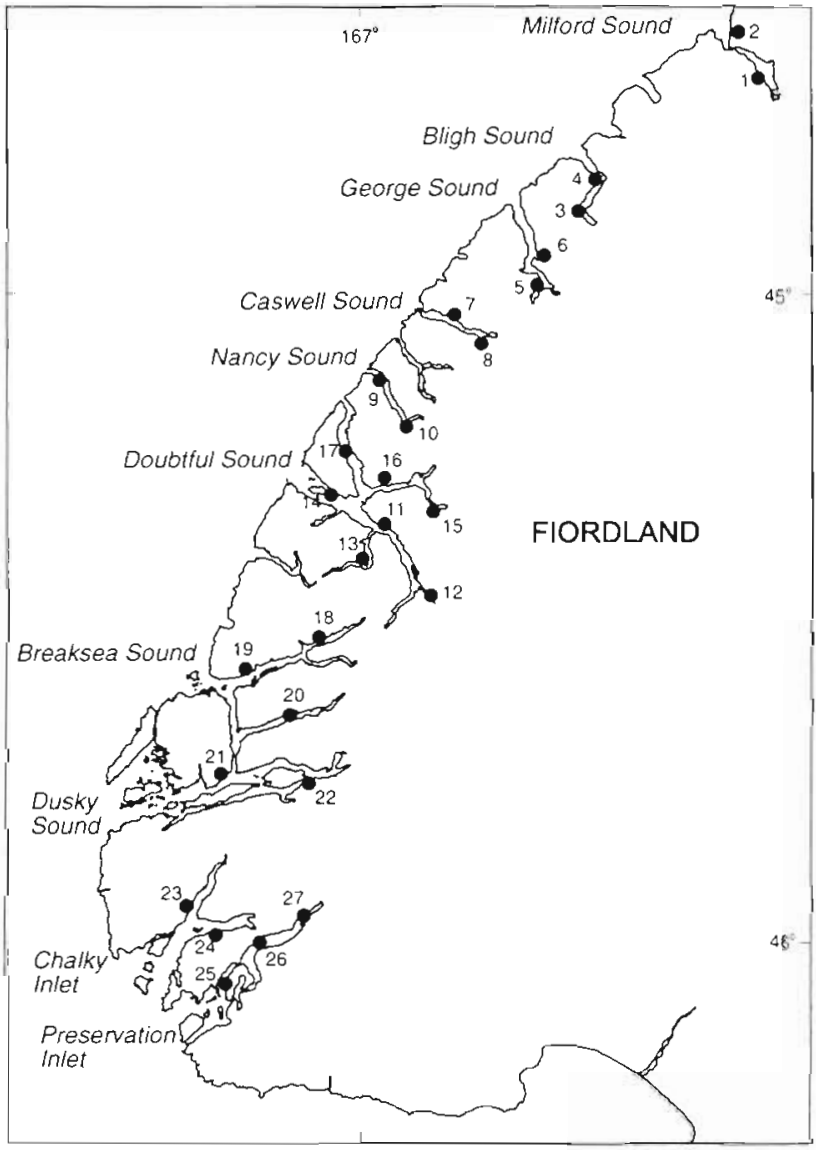

F-statistics were used to examine levels of genetic variance in Antipathes fiordensis populations, both between and within sites. $F_{S T}$ (the standardised genetic variation between populations) and $F_{I S}$ (the standardised genetic variation within populations) were calculated according to the methods of Weir \& Cockerham (1984) which account for differences in sample sizes among populations. Chi-squared tests were used to test the hypothesis of no genetic subdivision between populations (i.e. $F_{S T}=0$ ) and within populations (i.e. $F_{I S}=0$ ). Formulae used were from Waples (1987), where $\chi^{2}=2 N F_{S T}(k-1)$ with $(k-1)(s-1)$ degrees of freedom $(N=$ total number of individuals sampled, $k=$ number of alleles at a given locus, $s=$ number of populations) and $\chi^{2}=N\left(F_{I S}\right)^{2}(k-1)$ with $k(k-1) / 2$ degrees of freedom. Hierarchical analysis of $F_{S r}$ (Wright 1978) was used to partition the level of genetic variation within and among fiords.

An unbiased estimate of $F_{S T}$ and $95 \%$ confidence limits were calculated by jackknifing over loci (Weir 1991). These values were then used to determine the number of migrants per generation using the equation $N_{e} m=\left(1 / F_{S T}-1\right) / 4$ (Wright 1931), in order to estimate gene flow between Antipathes fiordensis populations. A second estimation of $N_{e} m$ was calculated according
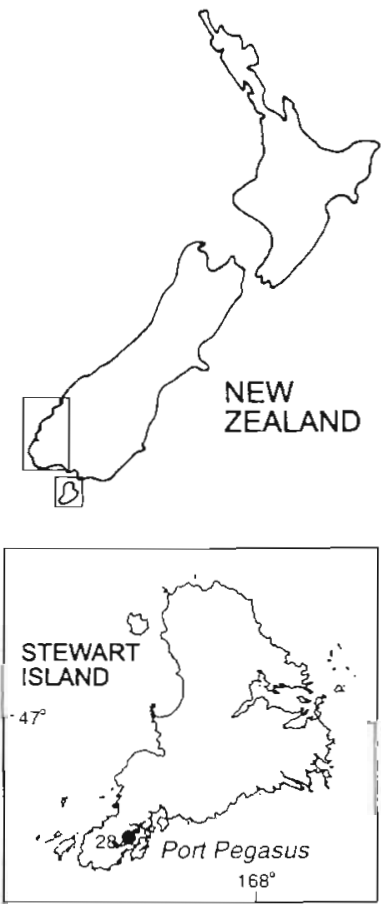

Fig. 1 Approximate locations of black coral study sites in Fiordland and at Stewart Island, New Zealand; $(\bullet$ collection sites

to the private allele method (Slatkin 1985, Barton \& Slatkin 1986).

Theories of isolation-by-distance (Wright 1943) predict that gene flow between populations will decrease with geographic distance and accordingly that genetic differentiation between populations will increase. This will be particularly true for species with restricted larval dispersal such as Antipathes fiordensis, provided equilibrium has been reached (Slatkin 1993). To test for evidence of isolation-by-distance in the Fiordland populations, pairwise levels of gene flow were regressed against geographic distance according to Slatkin (1993) using the programme DIST.

Observed genotypic diversity $\left(G_{0}\right.$, Stoddart 1983) was calculated at each site and compared with values expected under conditions of random sexual reproduction $\left(G_{E}{ }_{E}\right)$ in order to infer the relative contribution of sexual and asexual reproduction to recruitment in Antipathes fiordensis. For each population, genotypic diversity was calculated as $G_{0}=1 / \sum_{i=1}^{k} g_{i}^{2}$ where $g_{i}$ is the relative frequency of the $i$ th genotype and $k$ is the number of genotypes. This analysis included all genotypes and multiple clones. Expected genotypic diversity were calculated as $G^{*}{ }_{E}=1 / d+p / N$, where $d=\Sigma g_{i}^{2}$ for genotypes where $\left(g_{i} N\right)>1$, and $p=\sum g_{l}$ for geno- 
types where $\left(g_{1} N\right)<1$, and $N$ is the sample size (Stoddart 1983). As no genotypes were predicted to occur more than once in any population, $G_{E}^{*}$ was equal to $N$ in all populations.

\section{RESULTS}

\section{Genetic variation within populations}

Ten of the 12 loci scored were polymorphic, Est-1. Est-2, Idh, Pgm, Gpi, Mdh, Hk-1, Hk-2, La-1 and La-2.
Two loci, Me and Xo showed no allelic variation. The mean number of alleles per variable locus ranged between 1.7 and 2.8, with 60 to $100 \%$ of variable loci polymorphic at each site (Table 1).

All populations displayed genetic variation and some allele frequency differences were apparent among sites (Table 2). For example, Est-2 ${ }^{\circ} \mathrm{C}$ was present in $>70 \%$ of colonies sampled at Milford Site 2 and $>50 \%$ of colonies at Bligh Site 4 , but was generally rare elsewhere (Table 2). Similarly, Est-2 ${ }^{\circ}$ a was found in $44 \%$ of colonies at Chalky Site 23 , but represented $<10 \%$ of the sample at most other sites.

Table 1. Antipathes fiordensis. Genetic variation $( \pm \mathrm{SE})$ in black coral populations from Fiordland and Stewart Island, New Zealand. $N_{1}$ is the number of individual colonies at each site. $N_{C}$ is the number of unique genotypes observed at each site. $G_{0}$ and $G^{*}$ are observed and expected genotypic diversity, respectively. \% loci polymorphic is based only on the 10 polymorphic loci and does not include the 2 monomorphic loci screened

\begin{tabular}{|c|c|c|c|c|c|c|c|}
\hline \multirow[t]{2}{*}{ Site } & \multirow{2}{*}{$\begin{array}{l}\text { Mean no. of } \\
\text { alleles/locus }\end{array}$} & \multirow{2}{*}{$\begin{array}{c}\% \text { loci } \\
\text { polymorphic }\end{array}$} & \multicolumn{2}{|c|}{ Mean heterozygosity } & \multirow[t]{2}{*}{$N_{1}$} & \multirow[t]{2}{*}{$N_{C}$} & \multirow[t]{2}{*}{$G_{O}: G_{E}$} \\
\hline & & & Observed & $H-W$ expected & & & \\
\hline \multicolumn{8}{|c|}{ Milford Sound } \\
\hline Site 1 & $2.4(0.2)$ & 100 & $0.275(0.113)$ & $0.331(0.050)$ & 23 & 21 & 0.852 \\
\hline Site 2 & $2.0(0.2)$ & 90 & $0.304(0.096)$ & $0.378(0.066)$ & 30 & 28 & 0.834 \\
\hline \multicolumn{8}{|c|}{ Bligh Sound } \\
\hline Site 3 & $2.5(0.2)$ & 100 & $0.276(0.113)$ & $0.361(0.060)$ & 22 & 21 & 0.917 \\
\hline Site 4 & $2.4(0.2)$ & 100 & $0.387(0.114)$ & $0.422(0.068)$ & 30 & 29 & 0.938 \\
\hline \multicolumn{8}{|c|}{ George Sound } \\
\hline Site 5 & $2.4(0.2)$ & 90 & $0.327(0.101)$ & $0.380(0.067)$ & 30 & 27 & 0.833 \\
\hline Site 6 & $2.4(0.2)$ & 100 & $0.339\{0.107\}$ & $0.374(0.057)$ & 30 & 28 & 0.833 \\
\hline \multicolumn{8}{|c|}{ Caswell Sound } \\
\hline Site 7 & $2.6(0.2)$ & 100 & $0.348(0.116)$ & $0.402(0.053)$ & 30 & 29 & 0.937 \\
\hline Site 8 & $2.8(0.2)$ & 100 & $0.377(0.134)$ & $0.432(0.037)$ & 30 & 30 & 1.000 \\
\hline \multicolumn{8}{|c|}{ Nancy Sound } \\
\hline Site 9 & $1.7(0.2)$ & 60 & $0.320(0.121)$ & $0.287(0.073)$ & 15 & 10 & 0.555 \\
\hline Site 10 & $1.9(0.2)$ & 80 & $0.300(0.109)$ & $0.331(0.050)$ & 25 & 24 & 0.893 \\
\hline \multicolumn{8}{|c|}{ Doubtful Sound } \\
\hline Site 11 & $2.1(0.3)$ & 70 & $0.305(0.102)$ & $0.269(0.078)$ & 29 & 22 & 0.533 \\
\hline Site 12 & $1.9(0.3)$ & 60 & $0.369(0.121)$ & $0.313(0.089)$ & 13 & 13 & 1.000 \\
\hline Site 13 & $2.2\{0.2\}$ & 80 & $0.300(0.110)$ & $0.317(0.073)$ & 25 & 18 & 0.581 \\
\hline Site 14 & $2.0(0.3)$ & 70 & $0.358(0.116)$ & $0.310(0.075)$ & 25 & 19 & 0.609 \\
\hline Site 15 & $1.9(0.2)$ & 70 & $0.350(0.128)$ & $0.292(0.078)$ & 11 & 10 & 0.785 \\
\hline Site 16 & $2.1(0.2)$ & 80 & $0.350(0.122)$ & $0.305(0.073)$ & 25 & 12 & 0.269 \\
\hline Site 17 & $2.2(0.3)$ & 80 & $0.300(0.106)$ & $0.277(0.069)$ & 25 & 22 & 0.758 \\
\hline \multicolumn{8}{|c|}{ Dusky/Breaksea Sound } \\
\hline Site 18 & $2.6(0.2)$ & 100 & $0.361(0.109\}$ & $0.397(0.044)$ & 30 & 28 & 0.882 \\
\hline Site 19 & $2.8(0.2)$ & 100 & $0.298(0.099)$ & $0.414(0.041)$ & 29 & 27 & 0.879 \\
\hline Site 20 & $2.6(0.2)$ & 100 & $0.299(0.107)$ & $0.437(0.036)$ & 30 & 27 & 0.789 \\
\hline Site 21 & $2.7(0.2)$ & 100 & $0.261(0.108)$ & $0.349(0.049)$ & 30 & 29 & 0.937 \\
\hline Site 22 & $2.7(0.2)$ & 100 & $0.263(0.097)$ & $0.345(0.041)$ & 30 & 27 & 0.789 \\
\hline \multicolumn{8}{|c|}{ Chalky Inlet } \\
\hline Site 23 & $2.6(0.2)$ & 100 & $0.244(0.104)$ & $0.412(0.051)$ & 30 & 27 & 0.789 \\
\hline Site 24 & $2.5(0.2)$ & 100 & $0.287(0.109)$ & $0.336(0.063)$ & 30 & 27 & 0.714 \\
\hline \multicolumn{8}{|c|}{ Preservation Inlet } \\
\hline Site 25 & $2.4(0.2)$ & 100 & $0.268(0.085)$ & $0.323(0.061)$ & 30 & 25 & 0.811 \\
\hline Site 26 & $2.2(0.2)$ & 90 & $0.250(0.099)$ & $0.352(0.065)$ & 28 & 26 & 0.875 \\
\hline Site 27 & $2.7(0.2)$ & 100 & $0.244(0.098)$ & $0.407(0.050)$ & 29 & 28 & 0.935 \\
\hline \multicolumn{8}{|c|}{ Stewart Island } \\
\hline Site 28 & $2.3(0.2)$ & 100 & $0.300(0.104)$ & $0.413(0.042)$ & 20 & 19 & 0.909 \\
\hline
\end{tabular}


Table 2. Antipathes fiordensis. Allele frequencies at 10 variable loci for black coral at 28 sites throughout Fiordland, New Zealand

\begin{tabular}{|c|c|c|c|c|c|c|c|c|c|c|c|c|c|c|c|}
\hline \multirow{2}{*}{\multicolumn{2}{|c|}{ Locus/allele }} & \multicolumn{2}{|c|}{ Milford Sound } & \multicolumn{2}{|c|}{ Bligh Sound } & \multicolumn{2}{|c|}{ George Sound } & \multicolumn{2}{|c|}{ Caswell Sound } & Nan & hcy Sound & & Doub & btful Sound & \\
\hline & & & & & & & & & & Site & 9 Site 10 & Site 1 & 11 Site & 12 Site 13 & Site 14 \\
\hline Est-1 & d & 0.905 & 0.839 & 0947 & 0.966 & 0.846 & 0.964 & 0.86 & 0.750 & 1.00 & 1.000 & 1.000 & 1.00 & 1.000 & 1.000 \\
\hline & $b$ & 0.095 & 0.161 & - & 0.034 & 0038 & - & 0.10 & 0.150 & - & - & - & - & - & - \\
\hline & $r$ & - & - & 0.053 & - & 0115 & 0.036 & 0.03 & 0.100 & - & - & & - & - & - \\
\hline Est-2 & $a$ & - & 0.036 & - & . & - & 0.018 & 0.05 & 0.033 & - & 0.271 & 0.136 & 0.46 & - & - \\
\hline & $b$ & 0.952 & 0.232 & 0.905 & 0.483 & 0360 & 0.750 & 0.58 & 0.750 & 0.90 & 0.729 & 0.818 & 0.50 & 1.000 & 1.000 \\
\hline & c & 0.048 & 0.732 & 0.095 & 0.517 & 0.420 & 0.232 & $0.3 \mathrm{f}$ & 0.217 & 0.10 & - & $0.04: 5 y$ & 0.03 & - & - \\
\hline $\int d h$ & d & 0.800 & 0.964 & 0.833 & 0.966 & 1000 & 0.893 & 0.9 & 0.759 & 1.00 & 0.958 & 1.000 & 1.00 & 0.861 & 0684 \\
\hline & b & 0.150 & 0.036 & 0.119 & - & & 0.107 & 0.06 & 0.103 & - & 0.042 & - & - & 0139 & 0.316 \\
\hline & c & 0.050 & - & 0.048 & 0034 & - & - & - & 0.138 & - & - & - & - & - & - \\
\hline Pgm & d & - & - & - & - & - & - & 0.03 & - & 0.15 & 0.104 & 0.04 & 45 & 0.278 & - \\
\hline & b & 0.405 & 0.446 & 0.476 & 0.397 & 0.407 & 0.393 & 0.50 & 0.400 & 0.65 & 0.604 & 0.523 & 0.46 & 0472 & 0.605 \\
\hline & c & 0.571 & 0.429 & 0.476 & 0.483 & 0.481 & 0.536 & 0.44 & 0.517 & 0.20 & 0.292 & 0.43 & 0.53 & 0.250 & 0.342 \\
\hline & $d$ & 0.024 & 0.125 & 0.024 & 0.103 & 0.111 & 0.071 & 0.01 & 0.067 & - & - & - & - & - & 0.053 \\
\hline & e & - & - & 0.024 & 0.017 & - & - & - & 0.017 & - & - & - & - & - & - \\
\hline$G p i$ & a & 0.500 & 0.446 & 0.500 & 0.483 & 0.407 & 0.464 & $0.4 t$ & 0.500 & 0.40 & 0.500 & 0.45 & 0.50 & 0.472 & 0.500 \\
\hline & $b$ & 0.500 & 0.554 & 0.500 & 0.517 & 0.593 & 0.536 & 0.53 & 0.500 & 0.60 & 0.500 & 0.54 & 0.50 & 0.528 & 0.500 \\
\hline$M d h$ & $a$ & 0.143 & 0.286 & 0.357 & 0.362 & 0.222 & 0.214 & 0.32 & 0.267 & 0.20 & 0.208 & 0.136 & 019 & 0.306 & 0.237 \\
\hline & $b$ & 0.857 & 0.714 & 0.643 & 0.638 & 0.778 & 0.786 & 0.67 & 0.733 & 0.80 & 0.792 & 0.86 & 0.80 & 0.694 & 0.763 \\
\hline & c & - & - & - & - & - & - & - & - & - & - & - & - & - & - \\
\hline$H k-1$ & $a$ & - & - & - & - & - & - & - & - & - & - & - & - & - & 0.079 \\
\hline & $b$ & 0.375 & 0.393 & 0.450 & 0483 & 0.481 & 0.463 & 0.51 & 0.483 & 0.50 & 0.333 & 0.40 & 0.38 & 0.361 & 0.474 \\
\hline & c & 0.050 & 0.143 & 0.050 & 0.103 & -- & - & 0.03 & 0.067 & - & - & 0.136 & 0.23 & 0.056 & - \\
\hline & $d$ & 0.575 & 0.464 & 0.500 & 0414 & 0.519 & 0.537 & 0.4 & 0.450 & 0.50 & 0.667 & 0.45 & 0.38 & 0.583 & 0.447 \\
\hline $\mathrm{Hk}-2$ & $a$ & 0.238 & 0.179 & 0.048 & 0.155 & 0.130 & 0.125 & 0.25 & 0.155 & 0.05 & - & 0.20 & 0.15 & 0.111 & 0.105 \\
\hline & $b$ & - & 0.036 & - & - & 0.019 & - & - & - & - & - & 0.04 & 0.19 & - & - \\
\hline & c & 0.762 & 0.786 & 0.952 & 0.845 & 0.852 & 0.839 & 0.74 & 0.793 & 0.95 & 1.000 & 0.750 & 0.65 & 0.750 & 0.895 \\
\hline & $d$ & - & - & - & - & - & 0.036 & - & 0.052 & - & - & - & - & 0.139 & - \\
\hline La-1 & a & - & - & - & - & 0.037 & - & 0.07 & 0.103 & - & - & - & & 0.056 & - \\
\hline & b & 0.905 & 1.000 & 0.810 & 0.661 & 0.926 & 0.857 & 0.92 & 0.897 & 1.00 & 0.979 & 0.97 & 1.00 & 0.944 & 1.000 \\
\hline & c & 0.095 & - & 0.190 & 0.339 & 0.037 & 0.143 & - & - & - & 0.021 & $0.02:$ & - & - & - \\
\hline $\mathrm{La}-2$ & a & 0.024 & 0.018 & 0.071 & 0.259 & 0.259 & 0.179 & 0.08 & 0.100 & - & - & - & - & 0056 & 0.132 \\
\hline & b & 0.810 & 0.589 & 0.619 & 0.397 & 0.370 & 0.357 & 0.63 & 0.650 & 1.00 & 0.583 & 1.000 & 1.00 & 0.861 & 0.711 \\
\hline & c & 0.167 & 0.393 & 0.310 & 0.345 & 0.370 & 0.464 & 0.27 & 0.250 & - & 0.417 & - & - & 0.083 & 0.158 \\
\hline $\mathrm{n}$ & & 21 & 28 & 21 & 29 & 27 & 28 & 29 & 30 & 10 & 24 & 22 & 13 & 18 & 19 \\
\hline & & Doubt & ful Souns & d (cont.) & & Dusky/B & Breaksed & Sound & & Chalky & $y$ lnlet & Prese & ervation I & Inlet & wart lsland \\
\hline & & Site 15 & Site 16 & Site 17 & Site 18 & Site 19 & Site 20 & Site 21 & Site 22 & Site 23 & Site 24 & Site 25 & Site 26 & Site 27 & Site 28 \\
\hline Est-1 & $a$ & 1.000 & 1.000 & 1.000 & 0.778 & 0.815 & 0.808 & 0.931 & 0.889 & 0.852 & 0.963 & 0.940 & 0.846 & 0.885 & 0.867 \\
\hline & $b$ & - & - & - & 0.185 & $0.11 !$ & 0.192 & 0.034 & 0.074 & 0.111 & 0.037 & 0.060 & 0.154 & 0.077 & 0.133 \\
\hline & c & - & - & - & 0.037 & 0.07 .4 & - & 0.034 & 0.037 & 0.037 & - & - & - & 0.038 & - \\
\hline Est-2 & $a$ & - & 0.125 & 0.182 & 0.196 & 0.269 & 0167 & 0069 & 0.093 & 0.444 & 0.074 & 0.020 & 0.096 & 0.107 & 0.026 \\
\hline & $b$ & 0.900 & 0.875 & 0.773 & 0.804 & 0.673 & 0.759 & 0.897 & 0.833 & 0.426 & 0.667 & 0.980 & 0.673 & 0.661 & 0.711 \\
\hline & c & 0.100 & - & 0.045 & - & 0.058 & 0.074 & 0.034 & 0.074 & 0.130 & 0.259 & - & 0.231 & 0.232 & 0.263 \\
\hline$I d h$ & $a$ & 0.900 & 0.917 & 0.909 & 0.768 & 0.741 & 0.815 & 0.732 & 0.889 & 0.769 & 0.885 & 0.920 & 0.962 & 0.889 & 0.868 \\
\hline & $b$ & 0.100 & 0.083 & 0.091 & 0107 & 0.222 & 0148 & 0.196 & - & 0.115 & 0.019 & 0.080 & - & 0.111 & - \\
\hline & c & - & - & - & 0.125 & 0.037 & 0.037 & 0.071 & 0.111 & 0.115 & 0.096 & - & 0.038 & - & 0.132 \\
\hline Pgm & a & 0.100 & 0.083 & - & 0.018 & 0.037 & - & - & 0.056 & - & 0.01 .9 & - & - & - & - \\
\hline & $b$ & 0.600 & 0.458 & 0.455 & 0.500 & 0.556 & 0.444 & 0.431 & 0.704 & 0.611 & 0.574 & 0.380 & 0.500 & 0.411 & 0.474 \\
\hline & $c$ & 0.300 & 0.458 & 0.500 & 0.464 & 0.389 & 0.481 & 0.534 & 0.222 & 0.389 & 0.389 & 0.600 & 0.481 & 0.536 & 0.526 \\
\hline & d & - & - & 0.045 & 0.018 & 0.019 & 0.074 & 0.034 & 0.019 & - & 0.019 & 0.020 & 0.019 & 0.054 & - \\
\hline & $e$ & - & - & - & - & - & - & - & - & - & - & - & - & - & - \\
\hline$G p 1$ & $a$ & 0.500 & 0.500 & 0.341 & 0.500 & 0.426 & 0.463 & 0.448 & 0.481 & 0.463 & 0.500 & 0.440 & 0.481 & 0.446 & 0.500 \\
\hline & $b$ & 0.500 & 0.500 & 0.659 & 0.500 & 0.574 & 0.537 & 0.552 & 0.519 & 0.537 & 0.500 & 0.560 & 0.519 & 0.554 & 0.500 \\
\hline Mdh & d & 0.250 & 0.292 & 0.250 & 0.214 & 0.185 & 0.296 & 0.224 & 0.315 & 0.074 & 0.074 & 0.100 & - & 0.107 & 0.342 \\
\hline & $b$ & 0.750 & 0.667 & 0.750 & 0.786 & 0.815 & 0.704 & 0.776 & 0.685 & 0.926 & 0.926 & 0.900 & 1.000 & 0.893 & 0.658 \\
\hline & c & - & 0.042 & - & - & - & - & - & - & - & - & - & - & - & - \\
\hline$H k-1$ & $d$ & - & - & 0.045 & - & - & - & - & - & - & - & - & - & - & - \\
\hline & b & 0.500 & 0.500 & 0.432 & 0.429 & 0.389 & 0.365 & 0.375 & 0.370 & 0.413 & 0.360 & 0.341 & 0.375 & 0304 & 0.368 \\
\hline & $c$ & 0.100 & 0.083 & 0.023 & 0.107 & 0.148 & 0.154 & 0.071 & 0.037 & 0.043 & - & 0.045 & - & 0.143 & 0.158 \\
\hline & $d$ & 0.400 & 0.417 & 0.500 & 0.464 & 0.463 & 0.481 & 0.554 & 0.593 & 0.543 & 0.640 & 0.614 & 0625 & 0.554 & 0.474 \\
\hline$H k-2$ & $d$ & 0.300 & 0.125 & 0.045 & 0.054 & 0.093 & 0.167 & 0.241 & 0.111 & 0.167 & 0.111 & 0.229 & 0.135 & 0148 & 0.184 \\
\hline & $b$ & - & $\overrightarrow{-}$ & - & - & - & - & - & - & - & - & - & - & - & - \\
\hline & c & 0.700 & 0.875 & 0.955 & 0.946 & 0.907 & 0.833 & 0.759 & 0.889 & 0.833 & 0.889 & 0.771 & 0.865 & 0.815 & 0.816 \\
\hline & d & - & - & - & - & - & - & - & - & - & - & - & - & 0.037 & - \\
\hline $\mathrm{La}-1$ & $a$ & - & - & - & - & 0.074 & 0.019 & 0.034 & 0.074 & 0.074 & - & 0.080 & - & 0.037 & 0.188 \\
\hline & b & 1.000 & 1.000 & 1.000 & 0.786 & 0.759 & 0.704 & 0.879 & 0.852 & 0.833 & 0.926 & 0.840 & 0.808 & 0.704 & 0.813 \\
\hline & $c$ & - & - & - & 0.214 & 0167 & 0.278 & 0.086 & 0.074 & 0.093 & 0.074 & 0.080 & 0.192 & 0.259 & - \\
\hline La. 2 & त & - & - & - & 0.036 & 0111 & 0.167 & 0.034 & 0.074 & 0.204 & 0.167 & 0.300 & 0.231 & 0.125 & 0.053 \\
\hline & $b$ & 1.000 & 0.875 & 0.909 & 0.696 & 0.778 & 0.685 & 0.897 & 0.769 & 0.574 & 0.556 & 0.580 & 0.538 & 0.625 & 0.684 \\
\hline & $c$ & - & 0.125 & 0.091 & 0.268 & 0.111 & 0.148 & 0.069 & 0.130 & 0.222 & 0.278 & 0.120 & 0.231 & 0.250 & 0.263 \\
\hline $\mathrm{n}$ & & 10 & 12 & 22 & 28 & 27 & 27 & 29 & 27 & 27 & 27 & 25 & 26 & 28 & 19 \\
\hline
\end{tabular}


Genotype frequencies at the 10 variable loci were consistent with random mating at only 5 of the 28 sites: Doubtful Sites 12, 15, 16, 17 and Nancy Site 9. Genotype frequencies at the remaining 23 sites did not match those expected under conditions of HardyWeinberg equilibrium, even after correction of significance levels for multiple simultaneous tests (data not shown). Significant heterozygote deficits were apparent for Est-1, Est-2,Idh, Hk-2, La-1 and La-2 and were approximately twice as common as heterozygote excesses which were recorded for Pgm, Gpi and Hk-1. Mdh was the only locus which conformed to HardyWeinberg equilibria at all 28 locations.

Values of $F_{I S}$ varied significantly from zero at 8 of the 10 variable loci, indicating structuring within sites (Table 3). These are likely to be associated with departures from panmixis and correspond to results from the exact tests as outlined above: significant positive values of $F_{I S}$ reflecting heterozygote deficiencies at Est-1, Est-2, Idh, Hk-2, La-1 and La-2 and significant negative values of $F_{I S}$ reflecting heterozygote excesses at Gpi and $H k-1$.

\section{Genetic structure between populations}

There was evidence of subdivision among the 28 populations of Antipathes fiordensis. Values of $F_{S T}$ were generally low (ranging between 0.003 and 0.164 ) although the mean $F_{S T}$ and the $F_{S T}$ values at 6 of the 10 loci varied significantly from zero (Table 3 ). Hierarchical $F$-statistic analyses indicate that most of the variation $(64 \%)$ occurs among sites within fiords, with only $35 \%$ of the genetic variation occurring among fiords (Table 3).

Estimates of gene flow $\left(N_{e} m\right)$ calculated by $F_{S T}$ and private allele methods gave very similar results (5.2 and 3.2 migrants per generation respectively; Table 3 ). These values are relatively low, suggesting limited gene flow occurs between sites. However, there was no evidence of isolation-by-distance among the fiord populations of Antipathes fiordensis and regression analysis indicated no significant correlation between gene flow and geographic distance $\left(\mathrm{r}^{2}=\right.$ 0.017; Fig. 2). Nei's unbiased genetic distance (D) between sites was also very low, ranging from 0 to 0.107, which reflects the genetic similarity among fiords. UPGMA cluster analysis based on Nei's $D$ divided sites into 6 main groups. Sites within fiords often fell within the same group; however, each of the 6 groups contained sites from fiords that were widely separated (Fig. 3). In addition, the most geographically distant population, Stewart Island, did not exhibit any allelic differences to (Table 2), nor did it cluster independently of, the fiord populations (Fig 3).
Table 3. Antipathes fiordensis. Summary results from F-statıstic estimates of within population variation $\left(F_{F S}\right)$ and between population variation $\left(F_{S T}\right)$ at 10 loci for black coral populations from Fiordland and Stewart Island. Significant results from $\chi^{2}$ tests: $\cdots p<0.001, \cdots p<0.01, " p<0.05$. Also shown are the unbiased estumate of $F_{S T}( \pm 95 \%$ confidence limits), the estimated number of migrants per generation between sites $\left(N_{i} m\right.$ ) calculated using $F_{S T}(1)$ and by the private alleles method (2), and results from hierarchical F-statistic analyses

\begin{tabular}{|c|c|c|}
\hline Locus & $F_{/ 4}$ & $F_{S T}$ \\
\hline Est-1 & $0.954 \cdots$ & $0.029^{\circ}$ \\
\hline Est-2 & $0.621 \cdots$ & $0.164 \cdots$ \\
\hline$I d h$ & $0.891^{\cdots}$ & $0.037^{\cdots}$ \\
\hline Pgm & -0.009 & 0.019 \\
\hline Gpi & $-0.854 \cdots$ & 0.003 \\
\hline$M d l i$ & 0.037 & $0.035^{\cdots}$ \\
\hline$H k-1$ & $-0.421 \cdots$ & 0.008 \\
\hline$H k-2$ & $0.388^{\cdots}$ & 0.016 \\
\hline $\operatorname{La}-1$ & $0.794 \cdots$ & $0.060^{\cdots}$ \\
\hline $\mathrm{La}-2$ & $0.725 \cdots$ & $0.094^{\cdots}$ \\
\hline Mean & $0.313 \cdots$ & $0.046^{\circ}$ \\
\hline Unbiased $F_{S T}$ & \multicolumn{2}{|c|}{$0.046( \pm 0.004)$} \\
\hline$N_{m} m(1)$ & \multicolumn{2}{|c|}{$5.2(5.7-4.8)$} \\
\hline$N_{e} m(2)$ & \multicolumn{2}{|c|}{3.2} \\
\hline \multicolumn{3}{|c|}{ Hierarchical F-statistics } \\
\hline & Variance & $F_{S T}$ \\
\hline Sites within fiords & 0.122 & 0.034 \\
\hline Sites within total & 0.196 & 0.053 \\
\hline Fiords within total & 0.074 & 0.020 \\
\hline
\end{tabular}

\section{Clonality}

Of the 734 Antipathes fiordensis colonies sampled, 480 unique 10-locus genotypes were found. The remaining 254 colonies had one of 78 clonal genotypes. Of the 78 clonal genotypes detected, 5 were represented by more than 10 colonies while a further 8 were found between 5 and 10 times in the entire

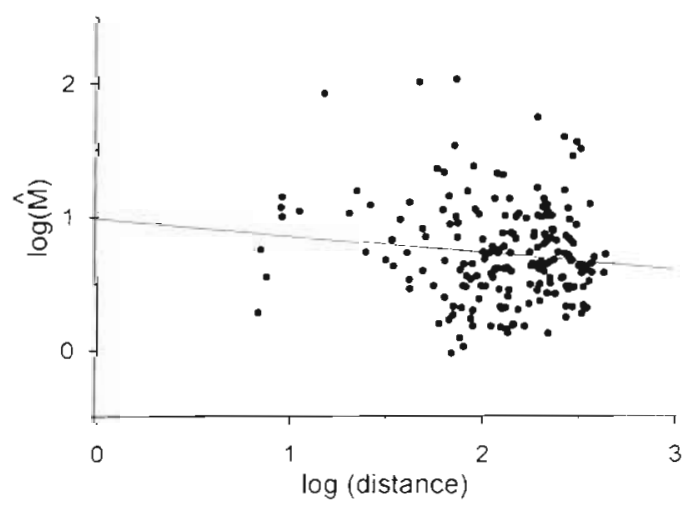

Fig. 2. Antipathes fiordensis. Plot of gene flow, $\log (\hat{N})$, against the log of geographic distance for black coral 
sample. Levels of genetic variability were moderate to high (Table 1) and the probability of these genotypes occurring more than once due to chance was calculated to be less than 1 in 1000 . Hence, colonies with identical 10-locus genotypes were considered to be the product of asexual reproduction.

Clonal genotypes were generally widespread, and colonies with the same genotype were found both within the same site, and in separate fiords. The ratio of observed to expected genotypic diversity was less than 1 (the expected value in a population reproducing exclusively by sexual means) at all but 2 sites (Caswell Site 8 and Doubtful Site 12; Table 1), although 21 of the other 26 sites had values of $G_{o}: G^{*}>0.7$ suggesting sexual reproduction contributed more to recruitment at these sites than asexual reproduction. Four sites had genotypic diversity ratios between 0.5 and 0.61 (Nancy Site 9, Doubtful Sites 11, 13, 14; Table 1) and the lowest value of $G_{0}: G_{E}{ }_{E}$ was 0.269 at Doubtful Site 16 (Table 1). At this site, only 7 of the 25 colonies sampled had unique genotypes and the remaining 18 colonies had only 5 clonal genotypes, one represented by 7 colonies, a second represented by 5 colonies and 3 other genotypes with 2 colonies each.

\section{DISCUSSION}

\section{Connectivity among fiords}

Significant genetic variation was found among Antipathes fiordensis populations throughout Fiordland. Interestingly, genetic differentiation was more apparent between sites within fiords rather than among fiords (Table 3). Values of $F_{S T}$ significantly more than zero, combined with a low migration rate
$\left(N_{e} m\right)$, suggest limited dispersal of A. fiordensis larvae between sites (i.e over distances of tens of kilometres) (see also Miller \& Grange in press). This result is consistent with our present knowledge of the limited mobility and short to moderate longevity of black coral larvae (Miller 1996) and with the restricted water movement within fiords (Stanton 1984). However there was no corresponding genetic differentiation over larger distances (i.e. among fiords), or between Fiordland and Stewart Island, as might be expected in a species with restricted larval dispersal. Furthermore there was no evidence to suggest any mechanisms are acting to produce isolation-by-distance in black coral populations (Fig. 3).

There are a number of possible interpretations of the observed population structure in Antipathes fiordensis. Genetic similarity among fiords may be maintained by larval exchange. Estimates of gene flow $\left(N_{0} m\right)$ suggest there will be an average of 3 to 5 migrants per generation among black coral populations. However,

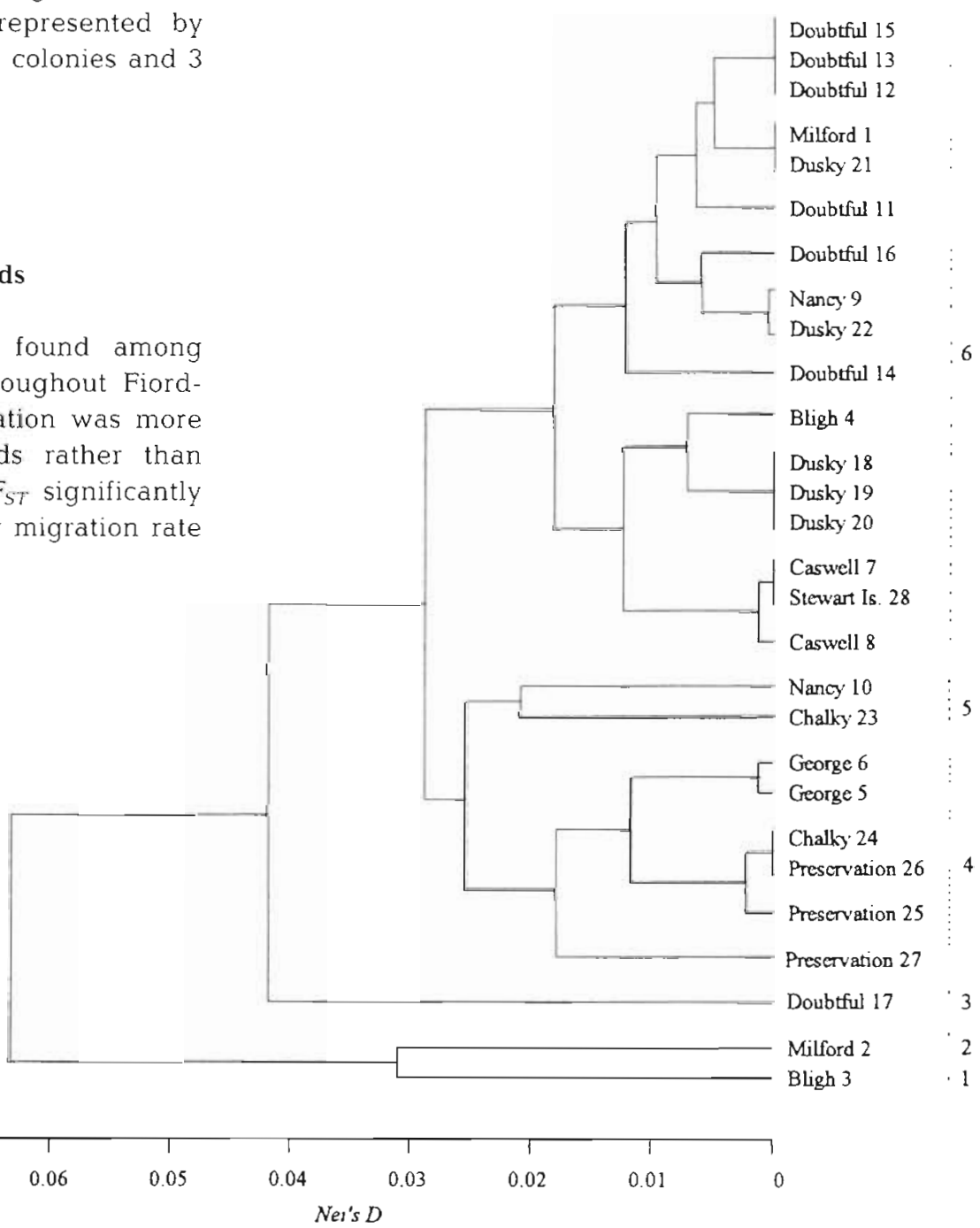

Fig. 3. Cluster analysis (UPGMA) of sites based on Nei's unblased genetic distance $(D)$. The 6 groups marked represent cluster levels at $D \approx 0.025$

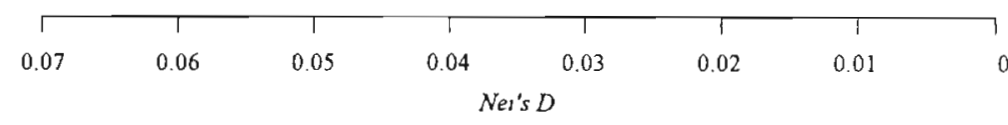


comparisons with $N_{e} m$ from studies of a variety of benthic marine invertebrates with pelagic larvae (e.g. scleractinian corals: $N_{t}, m=5.3$ to 16.4 . Stobart \& Benzie 1994, $N_{e} m=0.59$ to 4.81, Ayre \& Dufty 1994; zoanthids $N_{e} m=5.6$ to 35.5 , Burnett et al. 1995 ; sponges: $N_{e} m<$ 5. Benzie et al. 1994; clams: $N_{e} m \geq 20$, Benzie 1993) indicate $N_{\mathrm{e}} m$ in $A$. fiordensis is relatively low and that gene flow may be limited among sites. Furthermore, significant $F_{S T}$ values suggest there will be restricted dispersal of larvae (Table 3, Miller \& Grange in press). If larval dispersal does not occur even between sites within single fiords (i.e. $<15 \mathrm{~km}$ ) it seems unlikely that larvae would be dispersed outside of one fiord and into another. In addition, the net flow of seawater is into the fiords (Stanton 1978, 1984), so unless larvae are tolerant of low salinity and alter their buoyancy to become entrained within the outward flowing low salinity surface layer, they will most likely be retained within the natal fiord.

Genetic similarity among fiord populations of Antipathes fiordensis may be maintained by continual seeding from coastal populations living in deep water, or from sporadic larval dispersal from coastal populations during deep water renewal events (Stanton \& Pickard 1981). This theory assumes that deep water coral communities along the length of the Fiordland coast (covering a distance $>200 \mathrm{~km}$ ) comprise a single, genetically uniform population. It is thought that strong oceanic currents off the west coast of New Zealand form a large eddy spanning the length of the Fiordland coast (Chiswell 1996 and pers, comm.). This may promote panmixis in coastal populations of $A$. fiordensis, although analysis of samples from deep water outside the fiords will be necessary to confirm genetic relationships among these populations, and their relationship in terms of gene flow with the fiord populations

Genetic similarity between fiords may not necessarily reflect larval dispersal between them (e.g. Hedgecock 1982). It may be that fiord populations of Antipathes fiordensis are isolated by a combination of both physical and biological parameters, but that no differential selective pressures are operating which might cause genetic divergence. Variation in allelic frequencies occurs at some sites (e.g. Est $-2^{\circ} \mathrm{C}$ is very common at Milford Site 2 and Bligh Site 4 and Est $-2^{\circ} \alpha$ is common at Doubtful Site 12 but both Est-2 ${ }^{\circ} \mathrm{a}$ and Est$2^{\circ} \mathrm{C}$ are rare elsewhere; Table 2 ). It is unlikely that the rare alleles are common at these sites due to asexual reproduction as values of $G_{o}: G^{*}$ at the 3 sites are at least as high as values at the majority of sites sampled (Table 1). The small differences in allele frequencies may be the product of random genetic drift occurring between otherwise isolated sites. Migration rates $\left(N_{e} m\right)$ in $A$. fiordensis are $>1$, suggesting enough gene flow will occur between sites to prevent differentiation by genetic drift alone (Slatkin 1985, Slatkin \& Barton 1989). However, calculations of $N_{e} m$ are based on the assumption that the populations are at equilibrium (Wright 1965) and may be misleading for populations that are not (see below). Consequently, isolation and genetic drift cannot be ruled out as mechanisms underlying the genetic structuring of black coral populations.

Fiordland is a geologically recent habitat, and the genetic similarity among fiord populations of black coral are likely to be related to their relatively young age. As recently as 18000 yr ago, the sea level was $100 \mathrm{~m}$ lower than it is at present. The entrance sills of most of the fiords lie above this level (Stanton \& Pickard 1981) and at that time the majority of fiords would have been freshwater lake systems (Pickrill et al. 1992). The transition from fresh to marine habitats would have commenced around $12000 \mathrm{yr}$ ago, depending on the sill height of each fiord, to reach the present day sea level approximately 6500 yr ago. Black corals may live for hundreds of years (Grange 1985) and through asexual reproduction (Miller \& Grange in press), individual genotypes are potentially immortal. Thus because of overlapping generations which may be dominated by a few long-lived genotypes that contribute disproportionately to the reproductive output of the population (Grange 1985, in press), the genetic structure of Antipathes fiordensis populations may have changed little in the fiords since they were first seeded by coastal populations as the sea level rose (e.g. Potts 1984). The absence of any pattern of isolation-by-distance in a species with limited larval dispersal (Fig. 3) further suggests the fiord populations of $A$. fiordensis have not yet reached equilibrium following recent colonisation (Slatkin 1993). The genetic differentiation observed on smaller scales (i.e. between sites within fiords) may be an indication of wider scale divergence still to occur between isolated fiord populations, either from localised selection or genetic drift.

The results presented here for Antipathes fiordensis are unusual as the majority of studies of gene flow in marine invertebrates show genetic similarity across large distances only for species which have widely dispersed planktonic larvae (e.g. Hunt 1993, Williams \& Benzie 1993). In taxa with low dispersal larvae, genetic differentiation is generally apparent (e.g. Ayre et al. 1991. Hunt 1993, Burnett et al. 1995j. Few studies exist which report genetic similarity between geographically separated areas. However, a study of the deep water anemone Urticina eques, which has negatively buoyant crawling larvae, suggests genetic similarity in anemone populations separated by thousands of kilometres may also be attributable to the geologically 
recent nature of the seas sampled (Solé-Cava et al. 1994).

\section{Asexual reproduction}

The ratio of observed to expected genotypic diversity was $<1$ at 26 of the 28 sites studied (Table 1), indicating asexual reproduction occurs in Antipathes fiordensis throughout Fiordland. Furthermore, departures from Hardy-Weinberg equilibrium were apparent as both heterozygote deficiencies and excesses, which is indicative of asexual reproduction (e.g. Stoddart 1984, Ayre \& Willis 1988, Ayre et al. 1991).

The wide range of $G_{0}: G_{E}$ values $(0.296$ to 1.0 ; Table 1) is thought to reflect differential asexual recruitment among sites. However, the relatively high value of $G_{0}: G^{*}$ at most sites (>0.8; Table 1 ) confirms the conclusion of Miller \& Grange (in press), that sexual reproduction is the primary mode of reproduction and recruitment in Antipathes fiordensis.

The numerically abundant clones were individuals with genotypes largely comprised of common alleles, although the low probability of genotypes occurring more than once purely by chance suggests that most of these will result from asexual reproduction. However the wide distribution of some identical genotypes, particularly among fiords, remains unexplained. As discussed by Miller \& Grange (in press) the only observation of asexual reproduction in Antipathes fiordensis is a type of polyp 'bail-out' which produces large, highly mobile ciliated planulae. These planulae are likely to be more widely dispersed than the weak-swimming, negatively buoyant sexual larvae, although whether it is possible for asexual planulae to be transported between fiords is unknown. The occurrence of colonies with identical genotypes but of the opposite sex in different fiords (author's unpubl. data) suggests that some perceived clones may be sexual progeny, particularly given there is no evidence of hermaphroditism or sex change in antipatharians (Parker 1995). It is possible that historical patterns dating back to the initial colonisation of the fiords by $A$. fiordensis, combined with the longevity of individual colonies and the potential immortality of clonal genotypes, have resulted in the observed distributions of clonal genotypes.

Multiple clones found at the same site are often of the same size class and the same sex (author's unpubl. data). These observations suggest asexual recruitment to a site may occur in pulses, for instance resulting from regular cycles of asexual reproduction, or in response to environmental factors (see Miller \& Grange in press). Further studies will need to be undertaken to determine the exact nature and timing of asexual reproduction in Antipathes fiordensis.

\section{Conclusions}

Antipathes fiordensis populations display an unusual population genetic structure. Genetic differentiation is apparent on scales of tens of kilometres (between sites within fiords), which is consistent with the hypothesis that dispersal of $A$. fiordensis larvae is restricted by a combination of both biological and physical factors. However there is no evidence of isolation by distance, as may be expected in species with limited larval dispersal, and populations of $A$. fiordensis in geographically separated fiords remain genetically very similar. This pattern is suggestive of non-equilibrium populations and is likely to be related to the geologically young age of these populations in which little genetic divergence has taken place, despite restricted larval dispersal. This factor, coupled with widespread asexual reproduction and colony longevity is the most probable explanation of the observed genetic structure in A. fiordensis

Acknowledgements. Many thanks to Dick Singleton, Franz Smith, Mike Page, Ken Grange, Don Tindale, Lindsay Chadderton and the crew of MV 'Renown', for assistance with the field component of this study. Thanks also to the Department of Conservation who collected the coral samples from Stewart Island. Craig Mundy, Ken Grange, Geoff Chambers, Charlie Doherty and Jonathan Gardner provided many comments and suggestions on this manuscript. The genetic work for this study was done in the laboratories at the Institute for Molecular Systematics at Victoria University of Wellington, and was supported by a contract between NIWA and VUW Link.

\section{LITERATURE CITED}

Ayre DJ, Dufty S (1994) Evidence of restricted gene flow in the viviparous coral Seriatopora hystrix on Australia's Great Barrier Reef. Evolution 48:1183-1201

Ayre DJ, Read J, Wishart J (1991) Genetic subdivision within the eastern Australian population of the sea anemone Actinia tenebrosa. Mar Biol 109:379-390

Ayre DJ, Willis BL (1988) Population structure in the coral Pavona cactus: clonal genotypes show little phenotypic plasticity. Mar Biol 99:495-505

Barton NH, Slatkin M (1986) A quasi-equilibrium theory of the distribution of rare alleles in a subdivided population. Heredity 56:401-415

Benzie JAH (1993) Genetics of giant clams: an overview. In: Fitt WK (ed) The biology and mariculture of giant clams: a workshop held in conjunction with the 7 th International Coral Reef Symposium, 21-26 June 1992, Guam, USA. Proceedings No. 47, Australian Centre for International Agricultural Research, Canberra, p 7-13

Benzie JAH, Sandusky C, Wilkinson CR (1994) Genetic structure of dictyoceratid sponge populations on the western Coral Sea reefs. Mar Biol 119:335-345

Burnett WJ, Benzie JAH, Beardmore JA, Ryland JS (1995) Patterns of genetic subdivision in populations of a clonal cnidarian, Zoanthus coppingeri, from the Great Barrier Reef. Mar Biol 122:665-673 
Burton RS (1986) Evolutionary consequences of restricted gene flow among natural populations of the copepod Tigropus calufornicus. Bull Mar Sci 39:526-535

Chiswell SM (1996) Variabillty in the Southland Current, New Zealand. NZ J Mar Freshwat Res 30:1-17

Elston RC, Forthofer R (1977) Testing for Hardy-Weinberg equilibrium in small samples. Biometrics 33:536-542

Grange KR (1985) Distribution, standing crop, population structure, and growth rates of black coral in the southern frords of New Zealand. NZ J Mar Freshwat Res 19: $467-475$

Grange KR (1988) Redescription of Antipathes aperta, Totton. (Coelenterata: Antipatharia), an ecological dominant in the southern fiords of New Zealand. NZ J Zool 15:55-61

Grange KR (1990) Antipathes fiordensis, a new species of black coral (Coelenterata:Antipatharia) from New Zealand. NZ J Zool 17:279-282

Grange KR (1994) Coelenterata. In: McCrone A (ed) A draft status list for New Zealand's marine flora and fauna. Department of Conservation, Wellington, p 13-18

Grange KR (in press) Demography of black coral populations in Doubtful Sound, New Zealand: results from a sevenyear experiment. Proc 6th Int Conference on Coelenterate Biology

Grange KR, Singleton RJ (1988) Population structure of black coral, Antipathes aperta, in the southern fiords of New Zealand. NZ J Zool 15:481-489

Grange KR, Singleton RJ, Richardson JR, Hill PJ, Main WDeL (1981) Shallow rock wall biological associations of some southern fiords of New Zealand. NZ J Zool 8:209-227

Harrison PL, Wallace CC (1990) Reproduction, dispersal and recruitment of scleractinian corals. In: Dubinsky $\mathrm{Z}$ (ed) Coral reef ecosystems. Elsevier, Amsterdam, p 133-207

Hedgecock D (1982) Genetic consequences of larval retention: theoretical and methodological aspects. In: Kennedy VS (ed) Estuarine comparisons. Academic Press, New York, p 553-568

Hunt A (1993) Effects of contrasting patterns of larval dispersal on the genetic connectedness of local populations of two intertidal starfish, Patiriella calcar and $P$. exigua. Mar Ecol Prog Ser 92:179-186

Jackson JBC (1986) Modes of dispersal of clonal benthic invertebrates: consequences for species' distributions and genetic structure of local populations. Bull Mar Sci 39: $588-606$

Johnson MS, Threlfall TJ (1987) Fissiparity and population genetics of Coscinasterias calamaria. Mar I3iol 93:517-525

Knowlton N, Jackson JBC (1993) Inbreeding and outbreeding in marine invertebrates. In: Thornhill $N$ (ed) The natural history of inbreeding and outbreeding. University of Chicago Press, Chicago, p 200-249

Miller KJ (1996) Piecing together the reproductive habits of New Zealand's endemic black corals. Water and Atmosphere 4:18-19

Miller KJ, Grange KR (in press) Population genetic studies of the antipatharian black coral Antipathes fiordensis from Doubtful and Nancy Sounds, Fiordland, New Zealand. Proc 6th Int Conference on Coelenterate Biology

Miller RG (1966) Simultaneous statistical inference. McGrawHill, New York

Editorial responsibility: Otto Kinne (Editor), Oldendorf/Luhe, Germany
Parker NR (1995) Reproductive biology of black coral Antipathes fiordensis in Doubtful Sound, Fiordland. MSc thesis, University of Otago, Dunedin

Pickrill RA, Fenner JM, McGlone MS (1992) Late Quaternary evolution of a fiord environment in Preservation Inlet. New Zealand. Q Res 38:331-346

Potts DC (1984) Generation umes and the Quaternary evolution of reef-building corals. Palaeobiology 10:48-58

Slatkin M (1985) Rare alleles as indicators of gene flow. Evolution 39:53-65

Slatkin M (1993) Isolation by distance in equilibrium and nonequilibrium populations. Evolution 47:264-279

Slatkin M, Barton NH (1989) A companson of three indirect methods for estımating average levels of gene flow. Evolution 43:1349-1368

Sole-Cava AM, Thorpe JP, Todd CD (1994) High genetic similarity between geographically distant populations in a sea anemone with low dispersal capabilities. J Mar Biol Assoc UK 74:895-902

Stanton BR (1978) Hydrology of Caswell and Nancy Sounds. In: Glasby GP (ed) Fiord studies: Caswell and Nancy Sounds. New Zealand Oceanographic Institute Memoir 79. New Zealand Department of Scientific and Industrial Research, Wellington, p 73-82

Stanton BR (1984) Some oceanographic observations in the New Zealand fiords. Estuar Coast Shelf Sci 19:89-104

Stanton BR, Pickard GL (1981) Physical oceanography of the New Zealand Fiords. New Zealand Oceanographic Institute Memoir 88. New Zealand Department of Scientific and Industrial Research, Wellington

Stobart B, Benzie JAH (1994) Allozyme electrophoresis demonstrates that the scleractinuan coral Montipora digitata is two species. Mar Biol 118(2):183-190

Stoddart JA (1983) A genotypic diversity measure. J Hered $74: 489-490$

Stoddart JA (1984) Genetical structure within populations of the coral Pocillopora damicornis. Mar Biol 81:19-30

Swofford DL, Selander RB (1981) A FORTRAN program for the comprehensive analysis of electrophoretic data in population genetics and systematics. J Hered 72:281-283

Waples RS (1987) A multispecies approach to the analysis of gene flow in marine shore fishes. Evolution 41(2):385-400

Weir BS (1991) Genetic data analysis. Methods for discrete population genetic data. Sinauer Assoc, Sunderland, MA

Weir BS, Cockerham CC (1984) Estimating F-statistics for the analysis of population structure. Evolution 38(6) $1358-1370$

Williams ST, Benzie JAH (1993) Genetic consequences of long larval life in the starfish Linckia laevigata (Echınodermata: Asteroidea) on the Gireat Barrier Reef. Mar Biol 117:71-77

Wright S (1931) Evolution in Mendelian populations. Genetics, Princeton 16:97-159

Wright S (1943) Isolation by distance. Genetics, Princeton 28 : $114-138$

Wright S (1965) The interpretation of population structure by F-statistics with special regard to systems of matung. EvoIution 19:395-420

Wright S (1978) Evolution and the genetics of populations. University of Chicago Press, Chicago

Submitted: March 18, 1997, Accepted: October 14, 1997

Proofs received from author(s): December 17, 1997 